\title{
Effectiveness and side effects of anti-CD20 therapy for autoantibody-mediated blistering skin diseases:A comprehensive survey of 71 consecutive patients from the Initial use to 2007
}

\author{
Jennifer D Peterson' \\ Lawrence S Chan ${ }^{2,3,4}$ \\ 'Department of Dermatology, Texas \\ Tech University Health Sciences \\ Center at Lubbock, Lubbock, TX, USA; \\ ${ }^{2}$ Department of Dermatology; \\ ${ }^{3}$ Department of Microbiology/ \\ Immunology, University of Illinois \\ at Chicago, Chicago, IL, USA; \\ ${ }^{4}$ Medicine Service, Jesse Brown VA \\ Medical Center, Chicago, IL, USA
}

\begin{abstract}
In order to examine the efficacy and side effects of the monoclonal antibody anti-CD20 (rituximab) on autoimmune blistering skin diseases, we performed a comprehensive survey of 71 consecutive patients from initial use up to 2007, using the PubMed database. A heterogeneous group of patients, including 51 patients with pemphigus vulgaris, one with pemphigus vegetans, nine with pemphigus foliaceus, five with paraneoplastic pemphigus, four with epidermolysis bullosa acquisita, and one with both bullous pemphigoid and graft vs host disease was included in this survey. Overall the monoclonal antibody seems to be effective in that $69 \%$ of patients showed complete response, $25 \%$ of patients showed partial response, whereas $6 \%$ of patients showed progressive disease. Six deaths occurred in association with the treatment, with four of these deaths in patients with paraneoplastic pemphigus, a disease characteristically resistant to conventional medication and with a high mortality rate. Of note, 11 patients who received combined rituximab and intravenous immune globulin treatments had the best outcome: complete response without any serious side effects. Therefore further investigation on rituximab with controlled clinical trial is a worthy pursuit.
\end{abstract}

Keywords: blistering diseases, skin, anti-CD20, pemphigus, epidermolysis bullosa acquisita

\section{Introduction}

The anti-CD20 antibody, rituximab, which targets B cells, has been recently been used experimentally in patients affected by autoimmune blistering skin diseases, such as pemphigus and bullous pemphigoid. In the majority of reported cases, patients responded well; however, in a few cases, serious infections have resulted from the treatment. This article attempts to analyze the effectiveness, the potential side effects, and the precautionary measures the physicians should perform to minimize the serious infections or other side effects.

\section{Methods}

We performed a PubMed literature search utilizing rituximab including the following phrases in various combinations; pemphigus, pemphigoid, epidermolysis bullosa acquisita, bullous, and blistering. All case reports and studies were included in which rituximab was used to treat an autoimmune blistering disease. Articles excluded were those not written in English or providing a detailed abstract in English. Our literature search was performed at the end of August 2007, and therefore includes all articles present on PubMed at this time. We defined complete response to indicate resolution of all mucocutaneous lesions. Partial response was defined as greater than 50\% improvement of all mucocutaneous lesions. Progressive disease was defined as patients that showed less than $50 \%$ improvement 
of mucocutaneous lesions. We classified short-term adverse events as an event that occurred from the start of the treatment to the end of the treatment. Mid-term adverse events were identified as occurring up to six months post-treatment. Long-term adverse events are those adverse events that transpired from six months up to five years post-treatment.

\section{Results}

Of the 71 patients included in this review, 51 had pemphigus vulgaris, one had pemphigus vegetans, nine had pemphigus foliaceus, five had paraneoplastic pemphigus, four with epidermolysis bullosa acquisita, and one patient with simultaneous bullous pemphigoid and graft versus host disease. In regards to clinical response to rituximab, 49 (69.01\%) showed a complete response, 18 (25.35\%) showed partial response, and four $(5.63 \%)$ showed progressive disease (Table 1). Overall, 67 (94.37\%) of patients included in this review showed complete or partial clinical improvement. Of note, in the four patients with progressive disease, one had pemphigus foliaceus, one had pemphigus vegetans, and the other two had paraneoplastic pemphigus. The latter of these is characteristically resistant to all conventional treatments if the associated primary tumors can not be entirely removed.

There were six deaths total in association with rituximab usage: two short term, two mid term, and two long term. Of the deaths four had paraneoplastic pemphigus, one pemphigus vulgaris, and one bullous pemphigoid and graft versus host disease. Most causes of death were attributed to sepsis, congestive heart failure, or pneumonia. All cases of death involved patients treated with rituximab without combining intravenous immune globulin (IVIg). Complications from infection included four cases of sepsis or bacteremia (three short term and one long term), two cases of pneumonia (short term: pneumocystis pneumonia which led to death; mid term: community acquired pneumonia), and one case of infective arthritits (mid term due to Pseudomonas aeruginosa). A patient with paraneoplastic pemphigus developed ocular herpes simple virus (long term), varicella (long term), and Mycobacterium chelonae cutaneous infection (long term). Other short-term complications included atrial fibrillation with congestive heart failure which led to death in a patient with paraneoplastic pemphigus and deep venous thrombosis in a patient with epidermolysis bullosa acquisita. Of important note all complications, including death, were limited to 10 of the 71 patients.

\section{Discussion}

Rituximab is an anti-CD20 chimeric monoclonal antibody that targets pre-B cells, immature B cells, naïve B cells, and memory B cells. ${ }^{1}$ Plasma and stem cells lack CD20 and therefore are not targeted by rituximab., ${ }^{1,2}$ After binding of rituximab to $\mathrm{CD} 20+$ cells, cells undergo apoptosis via direct effect, complement and antibody dependent cytotoxicity, and inhibition of cell proliferation. ${ }^{2-4}$ Recovery of B cells begins 6-9 months after rituximab treatment, with levels returning to normal one year later. ${ }^{5}$ Kazkaz and colleagues, Sundharam, and Cooper and colleagues reported no autoantibody titers reduction during the treatment with rituximab, ${ }^{2,3,6}$ while Neidermeier and colleagues, Goebler and colleagues, and Herrmann and colleagues did report a decrease in antibody titers to desmoglein 3 and/or desmoglein 1. ${ }^{7-9}$ As noted by Antonucci, in the cases of complete response to rituximab, clinical improvement is not always associated with a decrease in autoantibody titers, which might be secondary to the different life spans of antibody producing plasma cells. ${ }^{10}$ In the small study by Arin and colleagues, some but not all patients experienced a decrease in antibody titers to desmoglein 1 and 3 associated with improvement in their disease..$^{5}$ In a child with pemphigus foliaceus, the clinical improvement by rituximab treatment was associated with reduction of anti-desmoglein 1 autoantibodies (from 1:1,280 to 1:16 after 7 courses of treatment). ${ }^{11}$ Interestingly, Marzano and colleagues reported that only those patients affected by pemphigus foliaceus, and not those affected by pemphigus vulgaris, showed reduction of anti-desmoglein autoantibody titer in parallel with clinical improvement. ${ }^{12}$ Niedermeier and colleagues also reported a patient with epidermolysis bullosa acquisita with complete remission after rituximab treatment, had clinical improvement which paralleled the decline of anti-basement membrane autoantibody titer. ${ }^{13}$ Furthermore, Ahmed and colleagues and Schmidt and colleagues reported reduction of autoantibody titers during the treatment with combined rituximab and IVIg. ${ }^{14,15}$ To what extent does the IVIg contribute to the autoantibody titer reduction is not clear and cannot be determined by the published data derived from this combined rituximab/IVIg study. ${ }^{14}$ In the previous studies of IVIg treatment for patients with pemphigus, it was reported that the autoantibody titers did reduce during the treatment. ${ }^{16}$

Rituximab was originally developed to treat refractory, low-grade, follicular, B cell non-Hodgkins lymphoma ${ }^{2}$, but has been used experimentally in various autoimmune diseases including autoimmune blistering disorders (pemphigus vulgaris, ${ }^{5-7,9-10,12,14,17-27}$ pemphigus vegetans, ${ }^{12}$ pemphigus foliaceus, ${ }^{5,8,11,12,28}$ paraneoplastic pemphigus, ${ }^{29-33}$ bullous pemphigoid, ${ }^{34}$ and epidermolysis bullosa acquisita ${ }^{13,35,36}$ ), systemic lupus erythematosus, Sjögren's syndrome, dermatomyositis, rheumatoid arthritis, myasthenia gravis, Wegner's 
Table I Survey data from 7I consecutive patients from the initial use of anti-CD20 therapy for autoantibody-mediated blistering skin diseases until 2007

\begin{tabular}{|c|c|c|c|c|c|c|c|c|}
\hline & Diagnosis & $\begin{array}{l}\text { Cycles } \\
\text { of rituximab }\end{array}$ & $\begin{array}{l}\text { Concomitant } \\
\text { meds }\end{array}$ & Response & $\begin{array}{l}\text { Short } \\
\text { term ae }\end{array}$ & $\begin{array}{l}\text { Mid } \\
\text { term ae }\end{array}$ & $\begin{array}{l}\text { Long } \\
\text { term ae }\end{array}$ & Ref \\
\hline I & PV & 1 & & CR & & & & 17 \\
\hline 2 & PV & I & & $\mathrm{CR}$ & & & & \\
\hline 3 & PV & I & & $C R$ & & & & \\
\hline 4 & PV & 1 & & $C R$ & & & & \\
\hline 5 & PV & 1 & & PR & & & & \\
\hline 6 & PV & 1 & & $C R$ & & & & \\
\hline 7 & PV & I & & $\mathrm{CR}$ & & & & \\
\hline 8 & PV & I & & $C R$ & & & & \\
\hline 9 & PV & I & & CR & & & & \\
\hline 10 & PV & 1 & & PR & & & & \\
\hline II & PF & 1 & & $C R$ & & & & \\
\hline 12 & $\mathrm{PF}$ & I & & PR & & & & \\
\hline 13 & PV & 2 , then mo & IVIG, NOS & $C R$ & & & & 14 \\
\hline 14 & PV & 2 , then mo & IVIG, NOS & $C R$ & & & & \\
\hline 15 & PV & 2 , then mo & IVIG, NOS & CR & & & & \\
\hline 16 & PV & 2 , then mo & IVIG, NOS & $C R$ & & & & \\
\hline 17 & PV & 2 , then mo & IVIG, NOS & $C R$ & & & & \\
\hline 18 & PV & 2 , then mo & IVIG, NOS & $\mathrm{CR}$ & & & & \\
\hline 19 & PV & 2 , then mo & IVIG, NOS & $C R$ & & & & \\
\hline 20 & PV & 2 , then mo & IVIG, NOS & $C R$ & & & & \\
\hline 21 & PV & 2 , then mo & IVIG, NOS & $C R$ & & & & \\
\hline 22 & PV & 3 , then mo & IVIG, NOS & $\mathrm{CR}$ & & & & \\
\hline 23 & PV & 4 , then mo & IVIG, NOS & $\mathrm{CR}$ & & & & \\
\hline 24 & PV & I, then q 2 wk & STpo, IVIG, MMF, CYP & PR & & & & 18 \\
\hline 25 & PV & 1 & STpo & PR & & & & 9 \\
\hline 26 & PV & 1 & STpo, CYP & $C R$ & & & & 19 \\
\hline 27 & PV & 1 & STpo, MMF & PR & & & & 6 \\
\hline 28 & PV & $\mathrm{I}$, then q mo & STpo, STiv, MMF & $\mathrm{CR}$ & & & & 20 \\
\hline 29 & PV & 2 & STpo,AZA & PR & & Pneumonia & & 21 \\
\hline 30 & PV & 2 & STpo, CYA, AZA & PR & & Infective & & \\
\hline & & & & & & arthritis & & \\
\hline 31 & PV & 1 & STpo & $C R$ & & & & \\
\hline 32 & PV & 1 & STpo & CR & & & & 22 \\
\hline 33 & PV & I & STpo, MMF & CR & & & & 7 \\
\hline 34 & PV & I & STpo & PR & & & & 23 \\
\hline 35 & PV & 1 & STpo & CR & & & & 24 \\
\hline 36 & PV & 1 & STpo, IVIG, MMF & $C R$ & & & & 15 \\
\hline 37 & PV & $\mathrm{I}$, then q 4-8 wk & STpo, & CR & & & & 25 \\
\hline 38 & PV & I & STpo, CYP & $\mathrm{CR}$ & & & & 26 \\
\hline 39 & PV & I & STpo, CYP & CR & Pneumonia, Death & & & \\
\hline 40 & PV & 1 & CYP & PR & & & & \\
\hline 41 & PV & 1 & & $C R$ & & & & 27 \\
\hline 42 & PV & I & & CR & & & & \\
\hline 43 & PV & 2 (I q year) & STpo & $C R$ & & & & 10 \\
\hline 44 & PV & 1 & STpo & $\mathrm{CR}$ & & & & \\
\hline 45 & PV & I & STpo & CR & & & & \\
\hline 46 & PV & 1 & STpo & $C R$ & & & & \\
\hline 47 & PV & I & STpo & $C R$ & & & & \\
\hline 48 & PV & I & STpo,AZA & $C R$ & & & & 5 \\
\hline 49 & PV & I & STpo,AZA & PR & & & & \\
\hline 50 & PV & I & STpo, MTX & PR & & & & \\
\hline 51 & PV & $\mathrm{I}$ & STpo, MTX & CR & & & & \\
\hline 52 & PF & 1 & STpo,AZA & CR & & & & \\
\hline 53 & PF & I & STpo, CYP, IVIG & PD & & & & 28 \\
\hline 54 & $\mathrm{PF}$ & I & STiv & $C R$ & & & & 8 \\
\hline 55 & PF & $q \mathrm{wk} \times 12$, then $\mathrm{q} 2 \mathrm{wk}$ & STpo & CR & & & & 11 \\
\hline
\end{tabular}


Table I (Continued)

\begin{tabular}{|c|c|c|c|c|c|c|c|c|}
\hline & Diagnosis & $\begin{array}{l}\text { Cycles } \\
\text { of rituximab }\end{array}$ & $\begin{array}{l}\text { Concomitant } \\
\text { meds }\end{array}$ & Response & $\begin{array}{l}\text { Short } \\
\text { term ae }\end{array}$ & $\begin{array}{l}\text { Mid } \\
\text { term ae }\end{array}$ & $\begin{array}{l}\text { Long } \\
\text { term ae }\end{array}$ & Ref \\
\hline 56 & PNP & I & STpo & PR & Bacteremia & & $\begin{array}{l}\text { Sepsis, HSV,VZV, M. } \\
\text { chenolae, Death }\end{array}$ & 29 \\
\hline \multirow[t]{2}{*}{57} & PNP & $\mathrm{I} / 2$ & STpo, CYP & PD & Death, Sepsis & & & 30 \\
\hline & & & & & AFIB, CHF, Death & & & \\
\hline 58 & PNP & $\mathrm{I}$, then q $2 \mathrm{wk}$ & STpo & PR & & & & 31 \\
\hline 59 & PNP & $\mathrm{I}$, then q 2 mos & STpo, CYP, DOX,VIN & PR & & & & 32 \\
\hline 60 & PNP & 3 & STpo, CYP,VIN & PD & & Death & & 33 \\
\hline 61 & $\mathrm{BP}+\mathrm{GVHD}$ & I & Anti-CD-25, STpo, MMF & CR & & & Death, Sepsis & 34 \\
\hline 62 & EBA & I & STpo,AZA, COL & $C R$ & DVT & & & 35 \\
\hline 63 & EBA & I & STpo, MMF & PR & & & & 36 \\
\hline 64 & EBA & I & STpo, IA, MMF & $C R$ & & & & 13 \\
\hline 65 & EBA & I & STpo, IA, MMF & $P R$ & & & & \\
\hline 66 & $\mathrm{PVg}$ & $\mathrm{I}$, then q mo & STpo & PD & & & & 12 \\
\hline 67 & PV & $\mathrm{I}, \mathrm{I} 0$ mos later $\mathrm{q} w \mathrm{k} \times 2$ & STpo & CR & & & & \\
\hline 68 & PV & 1 & STpo, CYP & $P R$ & & & & \\
\hline 69 & PF & I & & $C R$ & & & & \\
\hline 70 & PF & I & STpo & CR & & & & \\
\hline 71 & PF & 1 & STpo & $C R$ & & & & \\
\hline
\end{tabular}

Abbreviations: Anti-CD-25, anti-CD-25 antibody;AZA, azathioprine; COL, colchicines; CYA, cyclosporine A; CYP, cyclophosphamide; DAP, dapsone; DOX, doxorubicin; IVIG, intravenous immunoglobulin; IA, immunoabsorption; MMF, mycophenolate mofetil; MTX, methotrexate; NOS, Immunosuppressant not otherwise specified; PHO, extracorporeal photopheresis; PLA, plasmaphoresis; Stpo, steroids po; STiv, steroids intravenous; TS, trimethoprim/sulfamethazole;VIN, vincrsitine; CR, complete response; PR, partial response; PD, progressive disease; BP, bullous pemphigoid; EBA, epidermolysis bullosa acquisita; GVHD, graft versus host disease; PF, pemphigus foliaceus; PNP, paraneoplastic pemphigus; PV, pemphigus vulgaris; PVg, pemphigus vegetans; Ref, reference; ae, adverse effect associated with use of rituximab.

granulomatosis, ${ }^{1}$ idiopathic thrombocytopenic purpura, type II mixed cryoglobulinemia, ${ }^{37}$ autoimmune hemolytic anemia, IgM-associated polyneuropathies, pure red cell aplasia, and thrombotic thrombocytopenia purpura. ${ }^{4}$

Autoimmune blistering disorders are characterized by vesiculobullous eruptions affecting the skin and/or mucous membranes secondary to antibodies against cell surface antigens $^{1}$ pemphigus or basement membrane antigens (bullous pemphigoid ${ }^{34}$ and epidermolysis bullosa acquisita ${ }^{35}$ ). Although autoreactive $\mathrm{T}$ cells have been identified in some of these patients, the effector cells are autoreactive B cells by way of their tissue-specific autoantibodies. The pemphigus group has intraepidermal (flaccid) bullae and includes pemphigus vulgaris, pemphigus foliaceus, and paraneoplastic pemphigus. Pemphigus vulgaris is due to antibodies against desmoglein 3 (in the mucosal variant) or desmoglein 1 and 3 (in the mucocutaneous variant). Pemphigus foliaceus is a more superficial form of pemphigus with antibodies directed against only desmoglein $1 .{ }^{1}$ Finally, paraneoplastic pemphigus is a variant of pemphigus associated with non-Hodkins lymphoma, chronic lymphocytic leukemia, thymoma, or Castleman's disease and has a variety of target epithelial antigens. ${ }^{29,31}$ Paraneoplastic pemphigus carries a poor prognosis, with a 2 year $90 \%$ mortality rate. ${ }^{29}$ Bullous pemphigoid is characterized by tense, subepidermal, blisters of the skin due to antibodies against basement membrane antigens, bullous pemphigoid antigens 1 and 2. ${ }^{34}$ Epidermolysis bullosa acquisita is another subepidermal blistering disorder with antibodies against collagen VII, a component of the basement membrane zone, and is often recalcitrant to conventional treatment. ${ }^{35}$

The autoimmune blistering disorders mentioned above are all conventionally treated with topical corticosteroids along with systemic corticosteroids and/or other systemic immunosuppressants. Most patients respond favorably to conventional regimens; however, a minority of patients fails and other treatment options must be considered. Even for those patients who respond to the conventional treatment regimens of systemic corticosteroid and immunosuppressives, the side effects these patients encountered could be very substantial. ${ }^{38}$ Many patients developed steroid-induced diabetes, osteoporosis, pathologic fracture of bones, and serious infections. ${ }^{39,40}$ For these reasons, treatment options alternative to systemic corticosteroid and immunosuppressives are always sought by clinicians who care for this group of patients. The first reported use of rituximab was in a patient with follicular lymphoma-associated paraneoplastic pemphigus. ${ }^{32}$ Thereafter, reports emerged of rituximab used

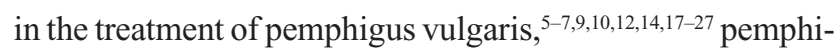
gus vegetans, ${ }^{12}$ pemphigus foliaceus, ${ }^{5,8,11,12,28}$ paraneoplastic pemphigus, ${ }^{29-33}$ bullous pemphigoid, ${ }^{34}$ and epidermolysis bullosa acquisita. ${ }^{13,35,36}$ 
Most commonly, rituximab is administered as a cycle of weekly infusions of $375 \mathrm{mg} / \mathrm{m}^{2}$ for four weeks. ${ }^{1}$ However, other infusion regimens have been described, including repeating a full cycle and/or monthly or every other week " $\mathrm{X}$ ". ${ }^{14,25,31}$ Concominant systemic therapy used during rituximab treatment have included prednisone, dexamethasone, mycophenolate mofetil, azathioprine, cyclophosphamide, colchicine, methotrexate, ${ }^{41}$ IVIg, plasmaphoresis, ${ }^{42}$ daclizumab (anti-CD25 antibody), ${ }^{34}$ and immunoabsorption. ${ }^{13}$

As noted earlier, in this review we found $69.01 \%$ of autoimmune blistering patients treated with rituximab showed a complete response and $25.35 \%$ showed partial response. Collectively, 67 (94.37\%) of patients showed clinical improvement. Of the patients with progressive disease despite rituximab therapy, one had pemphigus foliaceus, one had pemphigus vegetans, and the other two had paraneoplastic pemphigus. Although the exact duration of response of all reported patients treated with rituximab can not be accurately determined as many of these case reports contain a short follow-up time, we can safely state that current literatures indicate that the duration of response can last from at least 3 months ${ }^{23,32}$ to as long as 37 months. ${ }^{14}$ Furthermore, it is worthy to point out that the data from Ahmed studies suggest that combined IVIg and rituximab have the most promising effectiveness, resulting in complete response in all 11 treated patients (Table 1).

Most side effects secondary to rituximab occur at the time of infusion, are mild and transient, and include: headache ${ }^{41}$ fever, chills, nausea, shortness of breath, postural hypotension, ${ }^{1}$ pruritus, uticaria, ${ }^{37}$ and bronchospasm. ${ }^{18}$ These adverse events can be prevented and/or minimized with premedication of acetaminophen and diphenhydramine. ${ }^{1,18,41}$ Other side effects that have been encountered during rituximab usage for various diseases are as follows: Stevens-Johnson syndrome, toxic epidermal necrolysis, lichenoid dermatitis, vesiculobullous disease, vasculitis, acute respiratory distress syndrome, mycocardial infarctions, arrythmias, congestive heart failure, pneumonititis, tumor lysis syndrome, sepsis, hepatitis B reactivation with fulminant hepatitis, acute renal failure, anemia, lymphopenia, leukopenia, neutropenia, thrombocytopenia, ${ }^{37}$ meningoencephalitis, ${ }^{42}$ herpes zoster, varicella virus, Epstein Barr virus, and cytomegalovirus reactivation, pneumonia, ${ }^{43}$ and serum sickness. ${ }^{44}$ Additionally, progressive multifocal leukoencephalopathy has been reported in patients with systemic lupus erythematosus patients receiving treatment with rituximab. ${ }^{45}$ The most severe adverse event encountered in our review was death. A total of six deaths were associated with rituximab in these 71 patients. The distribution of the deaths as related to time was as follows; two short term, two mid term, and two long term. Of the patients that expired, four had paraneoplastic pemphigus, one pemphigus vulgaris, and one bullous pemphigoid and graft versus host disease. Most causes of death were attributed to sepsis, congestive heart failure, or pneumonia. It is also noteworthy that while these six bullous disease patients died while being treated with rituximab, these patients also had many confounding factors including underlying disease mortality, other preexisting diagnosis, and polypharmacy. Again, IVIg was not included in these six patients' therapeutic regimens.

While many authors reported that rituximab carries no increased risk of infection, ${ }^{17,18,22}$ the rituximab package insert does warn of an increase risk of infection and seven of the 71 patients included in this review had infections (Table 1). While a few bullous disease patients have developed serious infections on rituximab, these patients also had many confounding factors such as concomitant and past usage of immunosuppressants, underlying disease mortality (especially pemphigus vulgaris and paraneoplastic pemphigus), and other pre-existing diagnosis (for example, cancer or diabetes, which can cause immunosuppression) which have made them more susceptible to infections. To decrease the risk of infection while on rituximab it has been recommended to screen for hepatitis B virus, hepatitis $\mathrm{C}$ virus, and human immunodeficiency virus prior to treatment. Furthermore, close monitoring for signs of infection and prompt prescription of treatment for infection are essential. ${ }^{1,43}$ Rituximab should be avoided or used with extreme caution in autoimmune blistering disease patients with liver, kidney, neurological, or psychological disease, severe osteoporosis, immunodeficiency, active infections, history of cancer other than a lymphoproliferative disorder for which the patient might be receiving rituximab treatment with, and pregnant or lactating women, and used with cautiously in those with a history of cardiac disease such as angina and arrhythmias. ${ }^{1}$ Furthermore, long term consequences of rituximab are unknown and clinical trials are lacking. ${ }^{17}$

Although it can not be concluded from this small review, it is worthy noting that combined IVIg and rituximab treatment seems to have the least possibility of having serious infection (Table 1). In fact, none of the death has resulted in patients treated with combined rituximab and IVIg. A possible explanation is that the complete blockade of antibody production by anti-CD20 (rituximab) as a single treatment regimen to these patients may eliminate their abilities to defend against 
pathogens that are vulnerable to humoral immunity, therefore opening door for serious infections. However, the concomitant IVIg treatment could at least partially compensate for these patients' loss of humoral immunity by providing an externally produced antibody mixture, among them are antibodies specific for a variety of pathogens. Further, well-controlled studies are needed to determine if combination treatment of rituximab and IVIg leads to a decreased rate of infection, in comparison with treatment with rituximab alone.

\section{Conclusion}

In the analyses of these 71 patients treated with rituximab or combination of rituximab and IVIg, we found that $67(94.37 \%)$ of treated patients showed complete or partial clinical improvement. A total of six deaths were seen in the 71 patients; however, all patients had either a disease with an extremely poor prognosis (paraneoplastic pemphigus and graft versus host disease) or were exceptionally ill prior to rituximab administration (the pemphigus vulgaris patient). Infectious complications in autoimmune blistering patients treated with rituximab included sepsis, bacteremia, pneumonia, infective arthritis, herpes zoster, herpes simplex, and cutaneous Mycobacterium infection. Other noted side affects in our review included atrial fibrillation, congestive heart failure, and deep venous thrombosis. As mentioned early, these complications were limited to 10 of the 71 patients, and rituximab was well tolerated in the vast majority of patients. While, rituximab is a valuable treatment in autoimmune bullous diseases recalcitrant to other therapies, the risks of rituximab therapy must be weighed against the benefits on a case by case basis. Finally, the high rate of complete responses and the absence of serious side effects in a report of 11 patients treated with combined rituximab and IVIg should serve as an encouraging stimulus for a well-controlled clinical trial. However, the extremely high cost of combined rituximab and IVIg therapy may prove to be a road block for it to become a standard treatment option.

\section{Disclosure}

The authors report no conflicts of interest in this work.

\section{References}

1. Arin MJ, Hunzelmann N. Anti-B-cell-directed immunotherapy (rituximab) in the treatment of refractory pemphigus - an update. Eur J Dermatol. 2005;15:224-30.

2. Kazkaz H, Isenberg D. Anti B cell therapy (rituximab) in the treatment of autoimmune diseases. Curr Opin Pharmacol. 2004;4:398-402.

3. Sundharam J. Anti-CD20 monoclonal antibody (rituximab) in the treatment of pemphigus. Indian J Dermatol Venereol Leprol. 2006;72:173-4.
4. Virgolini L, Marzocchi V. Rituximab in autoimmune diseases. Biomed Pharmacother. 2004;58:299-309.

5. Arin MJ, Engert A, Krieg T, et al. Anti-CD20 monoclonal antibody (rituximab) in the treatment of pemphigus. Br J Dermatol. 2005;153:620-5.

6. Cooper HL, Healy E, Theaker JM, et al. Treatment of resistant pemphigus vulgaris with an anti-CD20 monoclonal antibody (rituximab). Clin Exp Dermatol. 2003;28:366-8.

7. Neidermeier A, Worl P, Barth S, et al. Delayed response of oral vulgaris to rituximab treatment. Eur J Dermatol. 2006;16:266-70.

8. Goebeler M, Herzog S, Brocker E-B, et al. Rapid response of treatment-resistant pemphigus foliaceus to the anti-CD20 antibody rituximab. Br J Dermatol. 2003;149:899-901.

9. Herrmann G, Engert A, Hunzelmann N. Treatment of pemphigus vulgaris with anti-CD20 monoclonal antibody (rituximab). Br J Dermatol. 2003; $18: 620-5$.

10. Antonucci A, Negosanti M, Tabanelli M, et al. Treatment of refractory pemphigus vulgaris with anti-CD20 monoclonal antibody (rituximab): five cases. J Dermatolog Treat. 2007;18:178-83.

11. Connelly EA, Aber C, Kleiner G, et al. Generalized erythrodermic pemphigus foliaceus in a child and its successful response to rituximab treatment. Pediatr Dermatol. 2007;24:172-6.

12. Marzano AV, Fanoni D, Venegoni L, et al. Treatment of refractory pemphigus with the anti-CD20 monoclonal antibody (rituximab). Dermatology. 2007;214:310-18.

13. Niedermeier A, Eming R, Pfütze M, et al. Clinical response of severe mechanobullous epidermolysis bullosa acquisita to combined treatment with immunoadsorption and rituximab (anti-CD20 monoclonal antibodies). Arch Dermatol. 2007;143:192-8.

14. Ahmed AR, Spigelman Z, Cavacini LA, et al. Treatment of pemphigus vulgaris with rituximab and intravenous immune globulin. $N$ Engl $J$ Med. 2006;355:1772-9.

15. Schmidt E, Herzog S, Brocker E-B, et al. Long-standing remission of recalcitrant juvenile pemphigus vulgaris after adjuvant therapy with rituximab. Br J Dermatol. 2005;153:449-51.

16. Sami N, Bhol KC, Ahmed RA. Influence of intravenous immunoglobulin therapy on autoantibody titers to desmoglein 3 and desmoglein 1 in pemphigus vulgaris. Eur J Dermatol. 2003;13:377-81.

17. Cianchini G, Corona R, Frezzolini A, et al. Treatment of severe pemphigus with rituximab: report of twelve cases and a review of the literature. Arch Dermatol. 2007;143:1033-8.

18. Salopek TG, Logsetty S, Tredget EE. Anti-CD20 monoclonal antibody (rituximab) for the treatment of recalcitrant, life-threatening pemphigus vulgaris with implications in the pathogenesis of the disorder. $J \mathrm{Am}$ Acad Dermatol. 2002;47:785-8.

19. Virgolini L, Marzocchi V. Anti-CD20 monoclonal antibody (rituximab) in the treatment of autoimmune diseases. Successful result in refractory pemphigus vulgaris: report of a case. Haematologica. 2003;88:ELT24.

20. Belgi AS, Azeez M, Hoyle C, et al. Response of pemphigus vulgaris to anti-CD20 antibody therapy (rituximab) may be delayed. Clin Exp Dermatol. 2005;31:143.

21. Dupuy A, Viguier M, Bedane C, et al. Treatment of refractory pemphigus vulgaris with rituximab (anti-CD20 monoclonal antibody). Arch Dermatol. 2004;140:91-6.

22. Espana A, Fernandez-Galar M, Lloret P, et al. Long-term complete remission of severe pemphigus vulgaris with monoclonal anti-CD20 antibody therapy and immunophenotype correlations. $J$ Am Acad Dermatol. 2004;50:974-6.

23. Wenzel J, Bauer R, Bieber T, et al. Successful rituximab treatment of pemphigus vulgaris resistant to multiple immunosuppressants. Acta Derm Venereol. 2005;85:185-6.

24. Cechhi R, Gasperini U. Severe pemphigus vulgaris treated with rituximab (mabthera). J Dermatol. 2005;32:862-4.

25. Kong HK, Prose NS, Ware RE, et al. Successful treatment of refractory childhood pemphigus vulgaris with anti-CD20 monoclonal antibody (rituximab). Pediatr Dermatol. 2005;22:461-4. 
26. Morrison LH. Therapy of refractory pemphigus vulgaris with monoclonal anti-CD20 antibody (rituximab). J Am Acad Dermatol. 2004;51:817-9.

27. Esposito M, Capriotti E, Gunta A, et al. Long-lasting remission of pemphigus vulgaris treated with rituximab. Acta Derm Venereol, 2006;86:87-9.

28. Johnston $\mathrm{S}$, Kennedy C. Images in clinical medicine. Pemphigus foliaceus. N Engl J Med. 2005;353:2589.

29. Barnadas MA, Roe E, Brunet S, et al. Therapy of paraneoplastic pemphigus with rituximab: a case report and review of literature. J Eur Acad Dermatol Venereol. 2006;20:69-74.

30. Schadlow MB, Anhalt GJ, Sinha AA. Using rituximab (anti-CD20 antibody) in a patient with paraneoplastic pemphigus. J Drugs Dermatol. 2003;2:564-7.

31. Van Rossum MM, Verhaegan NTM, Jonkman MF, et al. Follicular non-Hogkin's lymphoma with refractory paraneoplastic pemphigus: case report with review of novel treatment modalities. Leuk Lymphoma. 2004;45:2327-32.

32. Borradori L, Lombardi T, Samson J, et al. Anti-CD20 monoclonal antibody (rituximab) for refractory erosive stomatitis secondary to CD20+ follicular lymphoma-associated paraneoplastic pemphigus. Arch Dermatol. 2001;137:269-72.

33. Hoque SR, Black MM, Cliff S. Paraneoplastic pemphigus associated with CD20-positive follicular non-Hodgkin's lymphoma treated with rituximab: a third case resistant to rituximab therapy. Clin Exp Dermatol. 2007;32:172-5.

34. Szabolcs P, Reese M, Yancey KB, et al. Combination treatment of bullous pemphigoid with anti-CD20 and anti-CD25 antibodies in a patient with chronic graft-versus-host disease. Bone Marrow Transplant 2002;30:327-9.
35. Schmidt E, Benoit S, Brocker E-B, et al. Successful adjuvant treatment of recalcitrant epidermolysis bullosa acquisita with anti-CD20 antibody rituximab. Arch Dermatol. 2006;142:147-50.

36. Crichlow SM, Mortimer NH, Harman KE. A successful therapeutic trial of rituximab in the treatment of a patient with recalcitrant, high-titer epidermolysis bullosa acquisita. Br J Dermatol. 2007;156:194-6.

37. Scheinfeld N. A review of rituximab in cutaneous medicine. Dermatol Online J. 2006;12:3.

38. Sambrook PN. How to prevent steroid induced osteoporosis. Ann Rheum Dis. 2005;64:176-8.

39. Frediani B, Falsetti P, Baldi F, et al. Effects of 4-year treatment with once-weekly clodronate on prevention of corticosteroid-induced bone loss and fractures in patients with arthritis: evaluation with dual-energy x-ray absorptiometry and quantitative ultrasound. Bone. 2003;33:575-81.

40. Cranney A, Adachi JD. Corticosteroid-induced osteoporosis: a guide to optimum management. Treat Endocrinol. 2002;1:271-9.

41. Schmidt E, Hunzelmann N, Zillikens D, et al. Rituximab in refractory autoimmune bullous diseases. Clin Exp Dermatol. 2006;31:503-8.

42. El Tal AK, Posner MR, Spigelman Z, et al. Rituximab: a monoclonal antibody to CD20 used in the treatment of pemphigus vulgaris. $J \mathrm{Am}$ Acad Dermatol. 2006;55:449-59.

43. Corneley OA, Heidecke CN, Karthaus M. Opportunistic infections (OI) following monoclonal antibody treatment. 2005 ASCO Annual Meeting. J Clin Oncol. 2005;23(16 Suppl):2562.

44. Schutgens RE. Rituximab-induced serum sickness. Br J Haematol. 2006;135:147.

45. Rituxan. Rituxan (rituximab) package insert. Cited 2007 May 28 Available from http:/www.rituxan.com/. 
